\title{
Examining the Link Between Stress Level and Cybersecurity Practices of Hospital Staff in Indonesia
}

\author{
Muhammad Ali Fauzi* \\ muhammad.a.fauzi@ntnu.no \\ Norwegian University of Science and Technology \\ Gjøvik, Innlandet, Norway \\ Bian Yang* \\ bian.yang@ntnu.no \\ Norwegian University of Science and Technology \\ Gjøvik, Innlandet, Norway
}

\author{
Prosper Yeng* \\ prosper.yeng@ntnu.no \\ Norwegian University of Science and Technology \\ Gjøvik, Innlandet, Norway \\ Dita Rachmayani* \\ dh33ta@ub.ac.id \\ Universitas Brawijaya \\ Malang, Jawa Timur, Indonesia
}

\begin{abstract}
Since healthcare information systems have many important data that can attract many adversaries, it is important to take the right steps to prevent data breaches. Recent studies suggested that $85 \%$ of breaches involved a human element and the frequent patterns used are social engineerings. Therefore, many studies focus on making a better understanding of human behavior in cybersecurity and the factors that affect cybersecurity practices. However, there are only a few peer-reviewed studies that focus on the link between stress level and cybersecurity practices. In this study, we examined the link between stress level and cybersecurity practices among hospital employees in Indonesia by surveying 99 hospital workers. Perceived Stress Scale (PSS) was used to measure the employees' stress level and a new scale to measure hospital staff's risky cybersecurity practices was proposed. This study showed that both PSS and proposed cybersecurity practices scales are reliable with Cronbach's $\alpha$ value of more than 0.7 . The survey results also revealed that hospital worker's higher stress levels correlate significantly with riskier cybersecurity practices $\left(r_{s}=0.305, \mathrm{p}<0.01\right)$. Besides, a higher stress level is also significantly linked to certain cybersecurity practices, such as clicking on a link in an email from an unknown sender, not preventing colleagues from viewing patients' information for a non-therapeutic purpose, posting patient information on social media, ignoring colleagues who engage in negative information security practices, and failing to create strong passwords.
\end{abstract}

\section{CCS CONCEPTS}

- Security and privacy $\rightarrow$ Social aspects of security and privacy.

*All authors contributed equally to this research.

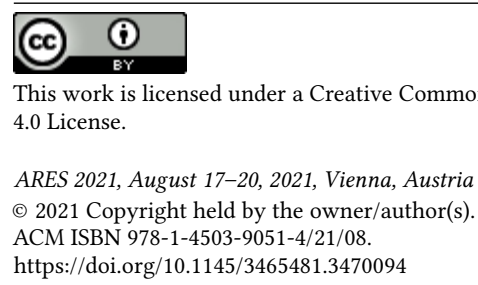

\section{KEYWORDS}

cybersecurity practices, behavior, stress, healthcare, hospital staff

\section{ACM Reference Format:}

Muhammad Ali Fauzi, Prosper Yeng, Bian Yang, and Dita Rachmayani. 2021. Examining the Link Between Stress Level and Cybersecurity Practices of Hospital Staff in Indonesia. In The 16th International Conference on Availability, Reliability and Security (ARES 2021), August 17-20, 2021, Vienna, Austria. ACM, New York, NY, USA, 8 pages. https://doi.org/10.1145/3465481.3470094

\section{INTRODUCTION}

The rise of digitalization in healthcare has a huge potential to improve patient care performance. However, it also carries a hazardous side-effect: healthcare system vulnerabilities. Cybercrime in healthcare can lead to not only data and financial loss but also medical devices and infrastructure damage [27]. Based on a recent investigations report [38], healthcare has become one of the top sectors that are in the biggest exposure of a data leak. The report also suggested that $85 \%$ of breaches involved a human element and around 35\% patterns in breaches are social engineering, the highest compared to any other patterns.

Humans are frequently referred to as the weakest link in cybersecurity [39]. No matter how sophisticated the technology is developed to improve cybersecurity systems, the system is still prone to be hacked due to human error [43]. For example, users that use a weak password, share their password with others, or forget to log out after using a public computer could lead to data breaches. Another real-life example is the compromising of personal information of Humana clients enrolled in the business's Medicare prescription medication coverage in 2006, which occurred after an insurance company employee accessed the data via a hotel computer and then neglected to delete the file [20]. Therefore, many studies focus on making a better understanding of human behavior in using computers and the internet and the factors that influence their cybersecurity practices. Whitty et al [42] studied the link between several factors including impulsivity, self-monitoring, and internal-external control and password sharing behavior. Halevi et al [15] evaluated the connection between cultural, personality, and demographic variables and cybersecurity practices. Gratian et al [12] investigated human characteristics such as personality traits, risk-taking preferences, and decision-making styles with cybersecurity behavior intentions. Yeng et al $[44,45]$ proposed a 
framework to analyze security practices of hospital employees that combine demographic and psycho-socio-cultural factors. Kennison and Chan-Tin [17] also studied how personality traits, risk-taking preferences, and secure password knowledge can be correlated to risky cybersecurity practices.

One of the factors that also have a significant effect on human behavior is their mental state such as their stress level. Research in psychology has shown that high stress level has a deleterious effect on decision making. Michailidis and Banks [23] found that employees who experienced burnout were more likely to make spontaneous or irrational decisions than those who felt more satisfied with their works. Another study [41] reported that stressed people will be slow in learning something new and likely choosing less profitable decisions. In healthcare, some researchers also examined the link between stress level and staff's performance especially related to patient safety $[25,36,40]$. However, these studies did not include cybersecurity practices.

In this study, we specifically examine the link between stress levels and cybersecurity practices among hospital workers in Indonesia. This work follows the hypothesis that hospital employees with higher stress levels have riskier security practices. This study aims for future use as basic data to include stress factors for promoting good cybersecurity behavior. Besides, this study also proposes a new scale to measure the cybersecurity practices of hospital staff.

\section{METHODOLOGY}

\subsection{Research approach}

As pictured in Figure 1, the goal of this study was to determine the influence of stress levels on hospital staffs' cybersecurity practices. An online questionnaire was used to collect data about healthcare staffs' demographic information, stress level, and cybersecurity practices in the last month. One month period is selected since the predictive validity of the Perceived Stress Scale (PSS), the scale used to measure stress level in this study, is expected to drop rapidly after four to eight weeks [7]. Since not all cybersecurity practices are carried out every day (e.g. updating, backup data, etc.), one month period is also considered ideal. Some prior researches also use one month period to collect user's cybersecurity practices $[14,29]$.

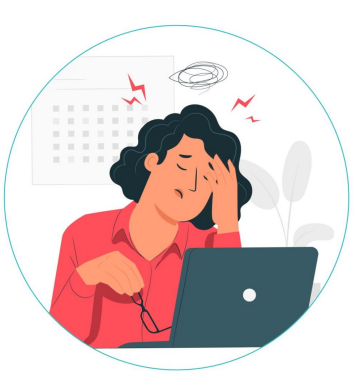

Stress Level

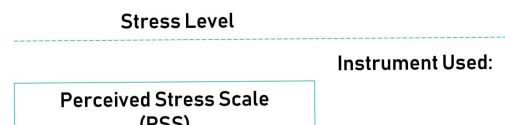
(PSS)

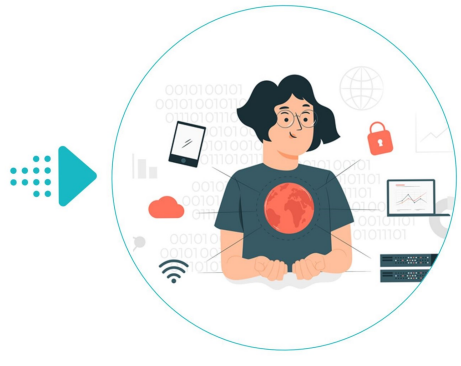

Cybersecurity Behavior

Hospital Staffs' Risky Cybersecurity Behavior Scale
Figure 1: Proposed approach.

\subsection{Participants and Procedures}

This study was conducted in a hospital in East Java, Indonesia and granted approval from the hospital's ethics committees. The participants of this study are recruited from the hospital's staff. Written consent was obtained electronically from all participants and questionnaires were completed and analyzed anonymously. In total, 112 participants completed the survey but 13 of them failed to answer an attention checking question correctly resulting in 99 qualified participants.

\subsection{Survey Instrument}

We developed a web-based questionnaire on Nettskjema, a tool for creating and administering online surveys managed by the University of Oslo [37]. Nettskjema ensures a high degree of security and privacy, which is very crucial for data collection. The questionnaire is in the Indonesian language and consists of three parts: demographic data, the stress level in the last month, and cybersecurity practices in the last month. Perceived stress scale (PSS) was used to measure respondents' stress levels in the last month while a new scale was proposed to measure hospital staff's risky cybersecurity practices. In addition, one attention-checking question was inserted in the middle of cybersecurity practices questions to make sure that the participants read the questions well. The question used a 5-point Likert scale ( 0 = disagree, 1 = slightly disagree, $2=$ neutral, $3=$ slightly agree, and $4=$ agree). The question text in English was as follows: This is an attention-checking question, select '2 (Neutral)' to show you really read this question. The participants who answer this question incorrectly are assumed to not read all of the questions well and will be disqualified.

2.3.1 Perceived Stress Scale (PSS). The instrument used to measure stress level in this study is the Perceived Stress Scale (PSS), the most common psychological instrument employed for measuring the perception of stress created by Cohen et al. [7]. It is a short and easy-to-use self-reported questionnaire established with acceptable psychometric properties to measure the extent to which situations in an individual's life are assessed as stressful. The PSS items examine the degree to which individuals feel about their life, specifically how unpredictable, uncontrollable, and overloaded they find their lives during the last month. Rather than concentrating on specific experiences or events, the items in the questionnaire are general in nature.

The PSS is available in three different versions. The original instrument is a 14 -item scale (PSS-14) with 7 positive and 7 negative items assessed on a 5-point Likert scale that was developed in English [7]. The PSS-14 was trimmed to ten questions (PSS-10) utilizing factor analysis based on data from 2,387 U.S. residents five years after its launch [6]. A four-item PSS (PSS-4) was also designed for scenarios requiring a very limited time or telephone interviews [6]. PSS-10 is the most widely used version. Based on the systematic review of the PSS psychometric evidence conducted by Lee [19], the 10-item PSS was found to have better psychometric qualities than the 14-item PSS, while the 4-item scale performed the worst. PSS-10 also have been translated into many languages other than English such as Greek[1], Arabic [5], Persian[22], Spanish[30], German[2], Korean[24], etc. Therefore, PSS-10 was used in this study. 
Since the study was administered in Indonesia, an Indonesian version of PSS-10 is used. This version has been tested and has a Cronbach Alpha coefficient value of 0.96 [28]. The possible scores ranging from $0-40$. Higher scores indicate that the respondent had a higher stress level in the last month.

2.3.2 Hospital Staff's Risky Cybersecurity Practices Scale. In this study, we propose a new scale to measure cybersecurity practices specifically for hospital staff. The hospital staffs' cybersecurity practices scale is developed partially based on the Human Aspects of Information Security Questionnaire (HAIS-Q) [26], Security Behavior Intentions Scale (SeBIS) [10]. Since these scales are designed for general computer users, we also conducted some interviews with 36 people including hospital staff and cybersecurity experts from several hospitals and universities in Indonesia, Ghana, and Norway to collect inputs and feedbacks about the scales. Then, based on their inputs and feedbacks, we modified the scale items to cover cybersecurity practices specifically for hospital staff. We were planning to use this scale to measure cybersecurity practices in hospitals in Indonesia, Ghana, and Norway. However, we only collect data from Indonesia for this study.

The scale asked participants to rate, on a scale of $0-4(0=$ disagree, 1 = slightly disagree, $2=$ neutral, $3=$ slightly agree, and $4=$ agree), how often they engaged in the specific practices in the last month. As displayed in Table 1, the final scale included 14 items with 11 items represent risky practices while only 3 items (item number 8, 13 , and 14) depict good practices. The possible scores ranging from 0-56 where the scores from the three good practices items were reversed. Higher scores indicate that the respondent had riskier cybersecurity practices in the last month.

\subsection{Reliability Testing and Correlation Analysis}

Since the proposed hospital staff's risky cybersecurity practices scale is new, a reliability analysis was performed by measuring Cronbach's $\alpha$ [8], one of the most popular measures of reliability in the social sciences [4]. Cronbach's $\alpha$ measures how closely related a set of items in the scale are as a group, on the survey result. Based on sources, Cronbach's $\alpha$ value of more than 0.7 is considered acceptable $[3,34,35]$. In addition, we also tested the reliability of the Indonesian version of PSS-10 using the same measure.

Meanwhile, in order to examine the link between the two scales (PSS and Hospital Staff's Risky Cybersecurity Practices Scale), Spearman's rank correlation coefficient $\left(r_{s}\right)$ [31] was used. It is one of the most widely used correlation measures in the psychology field [9].

\section{RESULT}

\subsection{General characteristics of participants}

In total, 112 staff (30.69\% of the population) participated in the survey but only 99 of them considered as qualified as they answered an attention-checking question correctly. The characteristic of participants is displayed in Table 2 while the population characteristic is displayed in Table 3. Generally, more females participated in this survey $(67.68 \%)$ than males $(32.32 \%)$. This gender distribution is
Table 1: Items for the Hospital Staffs' Cybersecurity Practices Scale

Item

1 In the last month, I usually write my user name and passwords on a piece of paper and stick the paper onto my computer for easy access

2 In the last month, I sometimes visit at least one of the following websites using the hospital's computer: social media; Dropbox and other public file storage systems; online music or videos sites; online newspapers and magazines; personal e-mail accounts; games; instant messaging services, etc

3 In the last month, I did not often read the alert messages/emails concerning security

4 In the last month, I sometimes click on a link in an email from an unknown sender

5 In the last month, I usually postpone software updating activities (restarting, clicking to run an update, accepting to update or follow update schedule) of my computers at my workplace

6 In the last month, I usually postpone backup activities when I am prompted

7 In the last month, I usually do not prevent my colleagues from seeing patients' records for a non-therapeutic purpose when I am working on a patients information on my laptop

8 In the last month, I did not post patient information on social media

9 In the last month, I sometimes share my passwords with my colleagues in hospital

10 In the last month, I usually do not take any action when I notice my colleague ignoring information security rules

11 In the last month, I usually talk about the patient condition in a shared patient ward in a hospital

12 In the last month, I usually dispose of sensitive personal health information (patients diagnosis and personal data) in the hospital

13 In the last month, I used a combination of letters, numbers, and symbols in my work passwords

14 In the last month, I have changed my passwords

similar to the population gender distribution. The age of participants varies from 21 until over 50 with about half of them aged between 31-40 (49.49\%). The percentage of participants with the age range $21-30$ becomes the second most with $31.31 \%$ while the proportion of participants aged between $41-50$ is $17.17 \%$. In the last place, participants aged over 50 contribute $2.02 \%$ of the total participants. Furthermore, by position, the majority of the participants are Nurse $(60.61 \%)$ and Pharmacy staff $(14.14 \%)$. The population also has a similar characteristic on the position as the majority of the workers are Nurse (44.11\%) and Pharmacy staff (8.22\%). From the position level, one of the hospital's executives participated in this survey. As expected, almost all of the participants are operational staff $(91,92 \%)$. Meanwhile, $7.07 \%$ of participants had manager and supervisor position levels. Concerning work experience, there are no participants with less than one year of work experience. The 
Table 2: Participant Characteristics

\begin{tabular}{|c|c|c|c|}
\hline Variable & Category & $\mathrm{n}$ & $\%$ \\
\hline \multirow[t]{3}{*}{ Gender } & $\mathrm{F}$ & 67 & $67.68 \%$ \\
\hline & M & 32 & $32.32 \%$ \\
\hline & Prefer not to say & 0 & $0.00 \%$ \\
\hline \multirow[t]{4}{*}{ Age } & $21-30$ & 31 & $31.31 \%$ \\
\hline & $31-40$ & 49 & $49.49 \%$ \\
\hline & $41-50$ & 17 & $17.17 \%$ \\
\hline & Over 50 & 2 & $2.02 \%$ \\
\hline \multirow[t]{8}{*}{ Position } & Top Level Management & 1 & $1.01 \%$ \\
\hline & Doctor & 3 & $3.03 \%$ \\
\hline & Nurse & 60 & $60.61 \%$ \\
\hline & Pharmachy staff & 14 & $14.14 \%$ \\
\hline & Nutritionist & 3 & $3.03 \%$ \\
\hline & Medical record staff & 5 & $5.05 \%$ \\
\hline & IT staff & 1 & $1.01 \%$ \\
\hline & Other & 12 & $12.12 \%$ \\
\hline \multirow[t]{3}{*}{ Position level } & Executive & 1 & $1.01 \%$ \\
\hline & Managers and supervisors & 7 & $7.07 \%$ \\
\hline & Operational staff & 91 & $91.92 \%$ \\
\hline \multirow[t]{7}{*}{ Work experience } & $<1$ Year & 0 & $0.00 \%$ \\
\hline & 1-5 Years & 22 & $22.22 \%$ \\
\hline & 6-10 Years & 26 & $26.26 \%$ \\
\hline & $11-15$ Years & 28 & $28.28 \%$ \\
\hline & 16-20 Years & 10 & $10.10 \%$ \\
\hline & 21-25 Years & 11 & $11.11 \%$ \\
\hline & $>25$ Years & 2 & $2.02 \%$ \\
\hline
\end{tabular}

Table 3: Population Characteristics

\begin{tabular}{llrr}
\hline Variable & Category & $\mathrm{n}$ & $\%$ \\
\hline Gender & $\mathrm{F}$ & 219 & $60.00 \%$ \\
& $\mathrm{M}$ & 146 & $40.00 \%$ \\
Position & Top Level Management & 3 & $0.82 \%$ \\
& Doctor & 23 & $6.30 \%$ \\
& Nurse & 161 & $44.11 \%$ \\
& Pharmachy staff & 30 & $8.22 \%$ \\
& Nutritionist & 22 & $6.03 \%$ \\
& Medical record staff & 6 & $1.64 \%$ \\
& IT staff & 1 & $0.27 \%$ \\
& Other & 119 & $32.60 \%$ \\
\hline
\end{tabular}

participant with 1-5 years, 6-10 years, and 11-15 years of work experience share a similar proportion with $22.22 \%, 26.26 \%$, and $28.28 \%$ respectively. In addition, $10.10 \%$ of participants had $16-20$ years of work experience, $11.11 \%$ of them had been working for $21-25$ years and $2.02 \%$ of them had experience of more than 25 years working in the hospital.

\subsection{PSS Score}

The distribution of the PSS scores is presented in Figure 2. The figure depicts a left-skewed distribution. It means that more people in a lower level of stress. The range of the PSS score is between 0-40 where higher scores indicate that the respondent had a higher stress level in the last month. The survey results show that the average of the participants' PSS score is 13.89 with a standard deviation (SD) of 4.41. The lowest score obtained is 3 (1 participant) while the highest score is 21 (4 participants).

In addition, we also conduct reliability testing of the PSS scale. Based on the survey results, the Cronbach $\alpha$ in this study for the PSS scale was 0.733 , suggesting that the items in the scale have relatively good internal consistency.

\subsection{Hospital Staffs' Cybersecurity Practices Score}

The statistic of the cybersecurity practices scores is presented in Figure 3 and Table 4. According to Figure 3, the score frequency distribution is left-skewed so that it is good news for the hospital as fewer employees have riskier cybersecurity practices. With regards to the possible score of $0-56$, the minimum score obtained is 0 (1 participant) while the highest score achieved is 31 (1 participant). Based on Table 1, the average score obtained is 16.37 with an SD of 7.66. On this scale, higher scores indicate that the respondent had riskier cybersecurity practices in the last month.

The score range of each item in the hospital employees' cybersecurity practices is between $0-4$. Table 4 shows that all of the items have a risky cybersecurity practices score average of less than 2 . Moreover, some of them have a score averaging less than 1. Item 2 becomes the one with the highest risk score average of 1.91. It means that more people visit external websites using the hospital's computer. Meanwhile, item 8 obtained the lowest risk score average of 0.38 . It means that almost all of the employees never post patient information on social media that can lead to personal information leakage. It is very reasonable because this practice is against the law.

Besides, we also tested the reliability of the hospital employees' cybersecurity practices scale using the survey results. The Cronbach's $\alpha$ for the scale in this study was 0.732 , indicating that the items in the scale had relatively good internal consistency. As displayed in Table 5, most of the items are significantly correlated with one another.

\subsection{Correlation Between Stress Level and Cybersecurity Practices}

The correlation between hospital staff's stress level and their cybersecurity practices is shown in Table 6. In general, stress level had a significant correlation with staff's cybersecurity practices $\left(r_{s}\right.$ $=0.305, \mathrm{p}<0.01)$. It means that employees with higher PSS scores tend to have higher risky cybersecurity practices scores. In other words, hospital staff with higher stress levels tend to practice riskier practices in terms of cybersecurity.

Furthermore, stress level has a significant correlation with several specific cybersecurity practices, namely item $4,7,8,10$, and 13 with correlation coefficient of $0.237(\mathrm{p}<0.005), 0.291$ ( $\mathrm{p}<0.01), 0.257$ $(\mathrm{p}<0.005), 0.308(\mathrm{p}<0.01)$, and $0.228(\mathrm{p}<0.005)$ respectively. The results suggest that there is a significant relationship between stress level and several cybersecurity practices in the items. Specifically, stress has a significant relationship with several risky practices including clicking on a link in an email from an unknown sender, not preventing colleagues from seeing patients' information, posting 


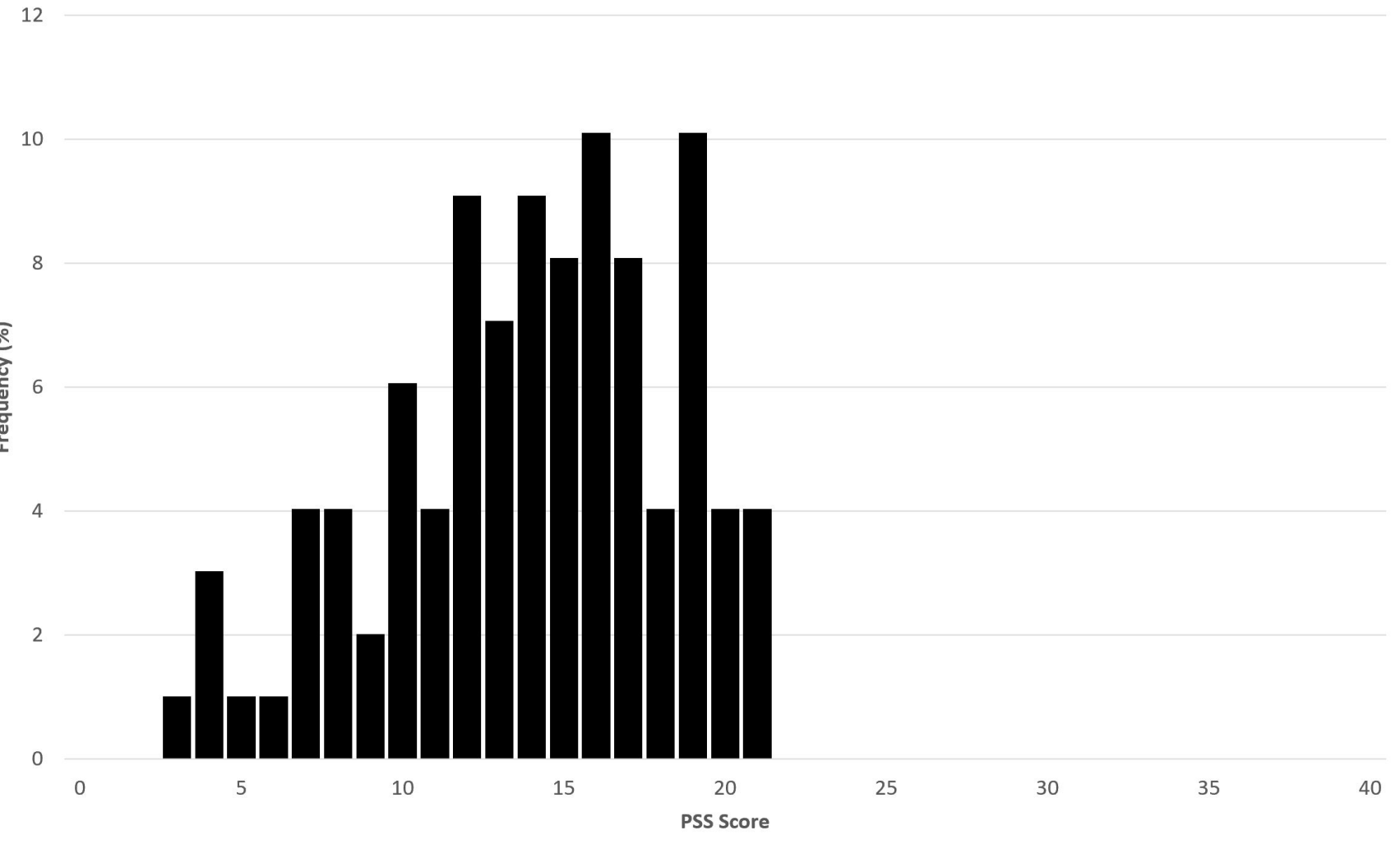

Figure 2: Frequency distribution of the hospital staffs' Perceived Stress Scale (PSS) scores.

Table 4: Means, standard deviations and ranges for the cybersecurity practices scale items.

\begin{tabular}{lccc}
\hline Item & Mean & SD & Range \\
\hline 1 & 1.30 & 1.46 & $0-4$ \\
2 & 1.91 & 1.37 & $0-4$ \\
3 & 1.68 & 1.27 & $0-4$ \\
4 & 0.89 & 1.08 & $0-4$ \\
5 & 2.00 & 1.03 & $0-4$ \\
6 & 1.73 & 1.05 & $0-4$ \\
7 & 0.63 & 1.13 & $0-4$ \\
8 & 0.38 & 1.15 & $0-4$ \\
9 & 0.87 & 1.38 & $0-4$ \\
10 & 1.09 & 1.34 & $0-4$ \\
11 & 0.60 & 1.36 & $0-4$ \\
12 & 1.49 & 1.79 & $0-4$ \\
13 & 1.01 & 1.67 & $0-4$ \\
14 & 1.68 & 1.89 & $0-4$ \\
Total Score & 16.37 & 7.66 & $0-56$ \\
\hline
\end{tabular}

patient information on social media, ignoring colleagues practicing bad information security practices, and not creating strong passwords. For other risky practices items in the survey, stress level also has a positive correlation, even though it is not significant, except for item 3. It means that higher stress levels also have a positive link with other risky practices even though it is not significant. Regarding item 3, unexpectedly, higher stress levels have a relationship with good practices in terms of reading the alert messages/emails concerning security. However, the relationship is very weak and not significant $\left(r_{s}=0.006\right)$.

\subsection{Principal Finding and Practical Application}

In this study, we looked at the links between stress levels and cybersecurity activities among Indonesian hospital personnel. Overall, we found evidence that stress is significantly associated with risky cybersecurity practices. This result agreed with many previous studies that reported a significant correlation between the stressful condition and decreased performance outcomes (e.g. [18, 25] etc.). In particular, stress correlates significantly with several specific cybersecurity practices, namely clicking on a link in an email from an unknown sender, not preventing colleagues from seeing patients' information, posting patient information on social media, ignoring colleagues practicing bad information security practices, and not creating strong passwords.

Acute stress affects our brains to consider reward and punishment in a way that can make us focus on pleasure and neglect the possible negative outcomes of our decisions [32, 33]. We are more likely to do things that feel good at the moment but are terrible for us in the long run when we are in a stressful condition. For example, when we are stressed and need to create a password for our new 


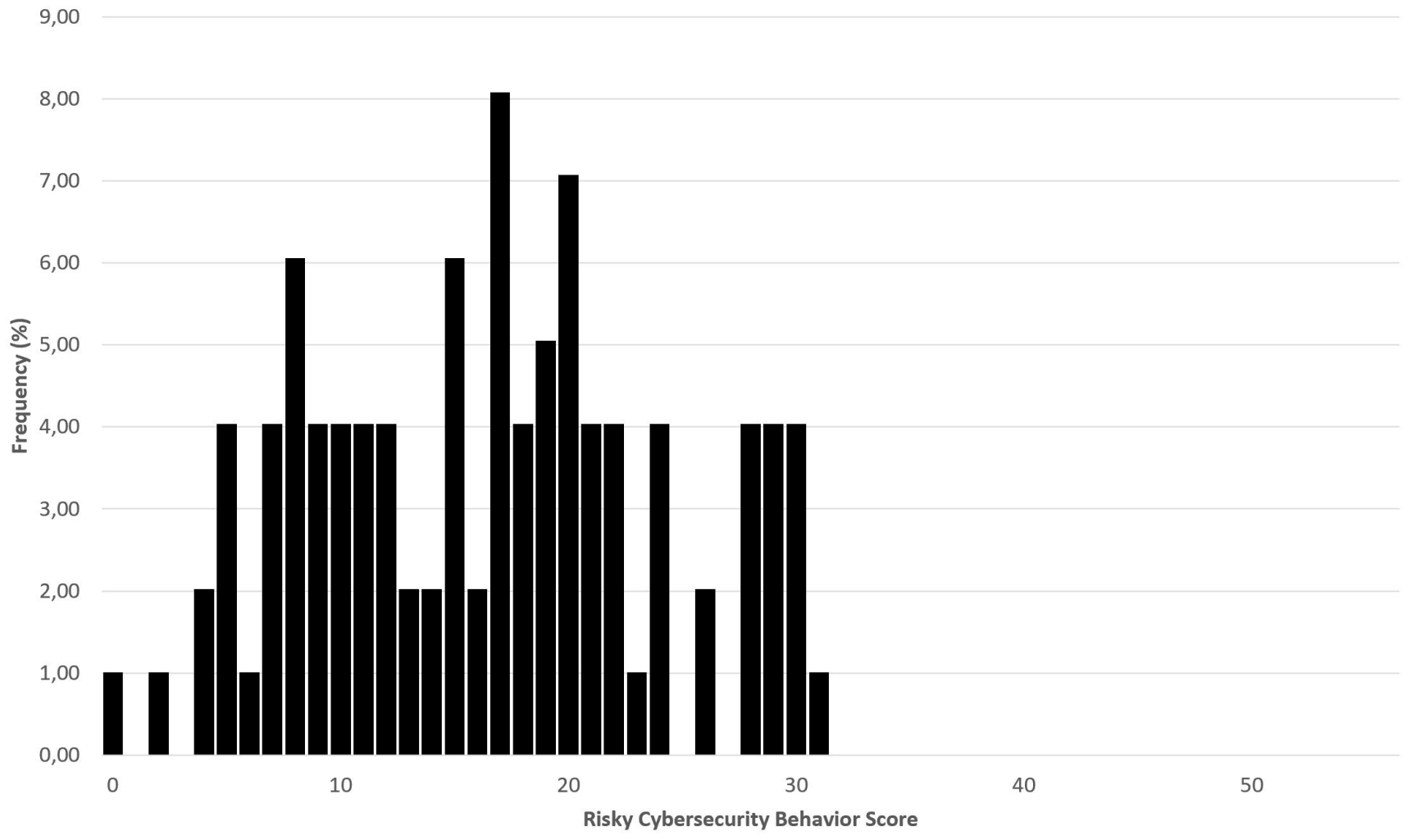

Figure 3: Frequency distribution of the hospital staffs' cybersecurity practices scores.

Table 5: Correlation of 14 items in the hospital staffs' cybersecurity practices scale

\begin{tabular}{|c|c|c|c|c|c|c|c|c|c|c|c|c|c|c|c|}
\hline & $\mathrm{I}_{1}$ & $\mathrm{I}_{2}$ & $\mathrm{I}_{3}$ & $\mathrm{I}_{4}$ & $\mathrm{I}_{5}$ & $\mathrm{I}_{6}$ & $\mathrm{I}_{7}$ & $\mathrm{I}_{8}$ & $\mathrm{I}_{9}$ & $\mathrm{I}_{10}$ & $\mathrm{I}_{11}$ & $\mathrm{I}_{12}$ & $\mathrm{I}_{13}$ & $\mathrm{I}_{14}$ & Total \\
\hline Item1 & 1.00 & - & - & - & - & - & - & - & - & - & - & - & - & - & - \\
\hline Item2 & 0.03 & 1.00 & - & - & - & - & - & - & - & - & - & - & - & - & - \\
\hline Item3 & 0.14 & 0.02 & 1.00 & - & - & - & - & - & - & - & - & - & - & - & - \\
\hline Item4 & $0.33^{* *}$ & 0.10 & 0.17 & 1.00 & - & - & - & - & - & - & - & - & - & - & - \\
\hline Item5 & -0.07 & 0.12 & 0.11 & 0.15 & 1.00 & - & - & - & - & - & - & - & - & - & - \\
\hline Item6 & 0.14 & 0.08 & 0.16 & $0.28^{* *}$ & $0.35^{* *}$ & 1.00 & - & - & - & - & - & - & - & - & - \\
\hline Item7 & $0.25^{*}$ & 0.14 & 0.13 & $0.38^{* *}$ & 0.05 & 0.18 & 1.00 & - & - & - & - & - & - & - & - \\
\hline Item 8 & $0.39^{* *}$ & 0.11 & 0.07 & $0.36^{* *}$ & 0.02 & 0.17 & $0.38^{* *}$ & 1.00 & - & - & - & - & - & - & - \\
\hline Item 9 & $0.43^{* *}$ & 0.13 & 0.12 & $0.39^{* *}$ & -0.13 & 0.11 & $0.35^{* *}$ & $0.29^{* *}$ & 1.00 & - & - & - & - & - & - \\
\hline Item 10 & 0.13 & -0.01 & 0.05 & $0.40^{* *}$ & 0.17 & 0.17 & $0.39^{* *}$ & $0.26^{*}$ & $0.49^{* *}$ & 1.00 & - & - & - & - & - \\
\hline Item 11 & $0.30^{* *}$ & 0.18 & 0.18 & $0.38^{* *}$ & 0.11 & $0.25^{*}$ & $0.58^{* *}$ & $0.40^{* *}$ & $0.54^{* *}$ & $0.44^{* *}$ & 1.00 & - & - & - & - \\
\hline Item12 & $0.23^{*}$ & 0.09 & 0.11 & $0.28^{* *}$ & 0.05 & $0.29^{* *}$ & $0.32^{* *}$ & 0.10 & $0.29^{* *}$ & $0.25^{*}$ & $0.30^{* *}$ & 1.00 & - & - & - \\
\hline Item 13 & 0.17 & 0.06 & 0.16 & $0.35^{* *}$ & $0.26^{*}$ & $0.22^{*}$ & $0.31^{* *}$ & $0.36^{* *}$ & 0.19 & $0.40^{* *}$ & $0.26^{*}$ & 0.19 & 1.00 & - & - \\
\hline Item 14 & 0.09 & -0.03 & $0.48^{* *}$ & 0.10 & $0.27^{* *}$ & 0.17 & 0.11 & 0.14 & 0.00 & $0.20^{*}$ & 0.06 & 0.05 & $0.58^{* *}$ & 1.00 & - \\
\hline Total & $0.51^{\text {** }}$ & $0.33^{* *}$ & $0.43^{* *}$ & $0.60^{* *}$ & $0.35^{* *}$ & $0.50^{* *}$ & $0.56^{* *}$ & $0.48^{* *}$ & $0.53^{* *}$ & $0.55^{* *}$ & $0.62^{* *}$ & $0.51^{* *}$ & $0.59^{* *}$ & $0.49^{* *}$ & 1.00 \\
\hline
\end{tabular}

${ }^{*} \mathrm{p}<0.005$.

${ }^{* *} \mathrm{p}<0.01$.

account, we are more likely to choose an easy-to-remember password or using the same password as for the other website. It makes us feel good because we will not be stressed to remember a new or complicated password even though we know that it may be compromised easily by some attackers in the future. In the same vein, a stressed person is more likely to access their social media during their work to search for joy. However, a stressed person tends to 
Table 6: PSS score correlation to cybersecurity practices score.

\begin{tabular}{ll}
\hline Item & Correlation coefficient $\left(r_{s}\right)$ \\
\hline Item 1 & 0.095 \\
Item 2 & 0.070 \\
Item 3 & -0.006 \\
Item 4 & $0.237^{*}$ \\
Item 5 & 0.167 \\
Item 6 & 0.109 \\
Item 7 & $0.291^{* *}$ \\
Item 8 & $0.257^{*}$ \\
Item 9 & 0.180 \\
Item 10 & $0.308^{* *}$ \\
Item 11 & 0.152 \\
Item 12 & 0.073 \\
Item 13 & $0.228^{*}$ \\
Item 14 & 0.155 \\
Total cybersecurity practices score & $0.305^{* *}$ \\
\hline${ }^{*} \mathrm{p}<0.005$. & \\
${ }^{* *} \mathrm{p}<0.01$. & \\
\hline
\end{tabular}

make errors and choose irrational decisions that possibly lead to posting sensitive data from work on social media. Furthermore, stress can make individuals feel exhausted, limited their attention and cognitive resources, and reduce their executive functioning $[16,32]$ so that they are more vulnerable to phishing attacks. Besides, these factors are also more likely to make the workers ignore their colleague's risky practices.

Regarding the practical application, this study contributes input for hospital management to concern about stress prevention among hospital workers as one of the factors to reduce risky cybersecurity practices. Our findings highlight the need of preventing stress in order to ensure appropriate cybersecurity practices and avoid negative consequences for the hospital such as data breaches. In addition, reducing hospital worker's stress levels can also contribute positively to their physical and mental health and work performance $[11,21]$.

\subsection{Limitation}

There are several limitations to our work. First, since a self-reported questionnaire was used to collect the data, even though anonymity is guaranteed, it is possible to have a desirability bias [13] where the participant may choose to answer in a way that they got low risky cybersecurity practices score or low stress level. Second, it is also possible that the user gave inaccurate answers because of fatigue, failures to understand questions, or even random clicking. In this study, we tried to minimize it by using an attention-checking question. Next, the result of this study may have been affected by selection bias. Employees with a high workload, which are more likely to have high stress levels, may have declined to participate due to limited time. As we can see from Figure 2, the stress level in our sample was relatively low. Finally, this study was crosssectional, hence, no conclusions about causal relationships can be formed.

\section{CONCLUSION}

Many hackers consider healthcare information systems as their target because of the abundance of important data contained in them. Therefore, it's crucial to take the proper precautions to avoid data breaches. In cybersecurity, humans are frequently referred to as the weakest link. According to recent reports, a large number of breaches featured a human element, with social engineering being the most common method exploited. As a result, many studies are devoted to gaining a better knowledge of human behavior in cybersecurity and the elements that influence cybersecurity practices. However, only a few research have looked into the relationship between stress and cybersecurity practices.

In this work, we surveyed 99 hospital workers in Indonesia to understand if there was a relationship between their stress levels and their cybersecurity practices. This study is based on the hypothesis that hospital staff who are under a lot of stress exhibit riskier security practices. The employees' stress levels were measured using the Perceived Stress Scale (PSS) and to assess hospital staff's risky cybersecurity practices, a new scale was proposed.

Based on the reliability analysis, both PSS and Hospital Staffs' Cybersecurity Practices scales have acceptable Cronbach's alpha values, which are 0.733 and 0.732 respectively. The survey also found that higher stress levels among hospital employees are significantly associated with riskier cybersecurity practices $\left(r_{s}=0.305\right.$, p 0.01). Furthermore, specifically, higher stress level also has a significant relationship with some specific cybersecurity practices, namely clicking on a link in an email from an unknown sender, not preventing colleagues from seeing patients' information, posting patient information on social media, ignoring colleague practicing bad information security practices, and not creating strong passwords.

The result of this study presents input for hospital management to give concern on stress prevention among their workers in order to reduce risky cybersecurity practices. Our findings highlight the need of preventing stress to ensure appropriate cybersecurity practices and avoid negative outcomes for the hospital such as data breaches.

For future work, stratifying the results by some characteristics such as age, gender, position, etc could give new perceptive and understanding in this topic. Comparative analysis from several countries with different cultures could also enrich our understanding of the relationship between stress levels and cybersecurity practices. Besides, since it was a cross-sectional study, no conclusions about causal relationships can be drawn. Therefore, analyzing the causal relationships between stress level and cybersecurity practices could be an important future work such as a randomized controlled trial in a lab-based environment or experiment sampling method.

\section{REFERENCES}

[1] Eleni Andreou, Evangelos C Alexopoulos, Christos Lionis, Liza Varvogli, Charalambos Gnardellis, George P Chrousos, and Christina Darviri. 2011. Perceived stress scale: reliability and validity study in Greece. International journal of environmental research and public health 8, 8 (2011), 3287-3298.

[2] Christina Diane Bastianon, Eva M Klein, Ana Nanette Tibubos, Elmar Brähler, Manfred E Beutel, and Katja Petrowski. 2020. Perceived Stress Scale (PSS-10) psychometric properties in migrants and native Germans. BMC psychiatry 20, 1 (2020), 1-9.

[3] J Martin Bland and Douglas G Altman. 1997. Statistics notes: Cronbach's alpha. Bmj 314, 7080 (1997), 572. 
[4] Douglas G Bonett and Thomas A Wright. 2015. Cronbach's alpha reliability: Interval estimation, hypothesis testing, and sample size planning. fournal of organizational behavior 36, 1 (2015), 3-15.

[5] Monique Chaaya, Hibah Osman, Georges Naassan, and Ziyad Mahfoud. 2010 Validation of the Arabic version of the Cohen Perceived Stress Scale (PSS-10) among pregnant and postpartum women. BMC psychiatry 10, 1 (2010), 1-7.

[6] Sheldon Cohen. 1988. Perceived stress in a probability sample of the United States. (1988).

[7] Sheldon Cohen, T Kamarck, and ROBIN Mermelstein. 1983. Perceived stress scale (PSS). J Health Soc Beh 24 (1983), 285.

[8] Lee J Cronbach. 1951. Coefficient alpha and the internal structure of tests. psychometrika 16, 3 (1951), 297-334.

[9] Joost CF de Winter, Samuel D Gosling, and Jeff Potter. 2016. Comparing the Pearson and Spearman correlation coefficients across distributions and sample sizes: A tutorial using simulations and empirical data. Psychological methods 21, 3 (2016), 273.

[10] Serge Egelman and Eyal Peer. 2015. Scaling the security wall: Developing a security behavior intentions scale (sebis). In Proceedings of the 33rd annual ACM conference on human factors in computing systems. 2873-2882.

[11] Tamara L Giluk. 2010. Mindfulness-based stress reduction: Facilitating work outcomes through experienced affect and high-quality relationships. (2010).

[12] Margaret Gratian, Sruthi Bandi, Michel Cukier, Josiah Dykstra, and Amy Ginther. 2018. Correlating human traits and cyber security behavior intentions. computers \& security 73 (2018), 345-358.

[13] Pamela Grimm. 2010. Social desirability bias. Wiley international encyclopedia of marketing (2010)

[14] LJ Hadlington. 2018. Employees attitudes towards cyber security and risky online behaviours: an empirical assessment in the United Kingdom. (2018).

[15] Tzipora Halevi, Nasir Memon, James Lewis, Ponnurangam Kumaraguru, Sumit Arora, Nikita Dagar, Fadi Aloul, and Jay Chen. 2016. Cultural and psychological factors in cyber-security. In Proceedings of the 18th International Conference on Information Integration and Web-based Applications and Services. 318-324.

[16] Karim S Kassam, Katrina Koslov, and Wendy Berry Mendes. 2009. Decisions under distress: Stress profiles influence anchoring and adjustment. Psychological science 20, 11 (2009), 1394-1399.

[17] Shelia M Kennison and Eric Chan-Tin. 2020. Taking Risks With Cybersecurity: Using Knowledge and Personal Characteristics to Predict Self-Reported Cybersecurity Behaviors. Frontiers in Psychology 11 (2020), 3030.

[18] Vicki R LeBlanc. 2009. The effects of acute stress on performance: implications for health professions education. Academic Medicine 84, 10 (2009), S25-S33.

[19] Eun-Hyun Lee. 2012. Review of the psychometric evidence of the perceived stress scale. Asian nursing research 6, 4 (2012), 121-127.

[20] Divakaran Liginlal, Inkook Sim, and Lara Khansa. 2009. How significant is human error as a cause of privacy breaches? An empirical study and a framework for error management. computers \& security 28, 3-4 (2009), 215-228.

[21] Corey S Mackenzie, Patricia A Poulin, and Rhonda Seidman-Carlson. 2006. A brief mindfulness-based stress reduction intervention for nurses and nurse aides. Applied nursing research 19, 2 (2006), 105-109.

[22] Saman Maroufizadeh, Armin Zareiyan, and Naseh Sigari. 2014. Reliability and validity of Persian version of perceived stress scale (PSS-10) in adults with asthma. Archives of Iranian medicine 17, 5 (2014), 0-0.

[23] Evie Michailidis and Adrian P Banks. 2016. The relationship between burnout and risk-taking in workplace decision-making and decision-making style. Work \& Stress 30, 3 (2016), 278-292.

[24] Jun O Park and Young Seok Seo. 2010. Validation of the perceived stress scale (PSS) on samples of Korean university students. Korean fournal of Psychology: General 29, 3 (2010), 611-629.
[25] Young-Mi Park and Souk Young Kim. 2013. Impacts of job stress and cognitive failure on patient safety incidents among hospital nurses. Safety and health at work 4, 4 (2013), 210-215.

[26] Kathryn Parsons, Dragana Calic, Malcolm Pattinson, Marcus Butavicius, Agata McCormac, and Tara Zwaans. 2017. The human aspects of information security questionnaire (HAIS-Q): two further validation studies. Computers \& Security 66 (2017), 40-51.

[27] Eric D Perakslis. 2014. Cybersecurity in health care. N Engl f Med 371, 5 (2014), 395-397.

[28] Tan Lee Pin. 2011. Hubungan Kebiasaan Berolahraga Dengan Tingkat Stres Pada Mahasiswa Fakultas Kedokteran Universitas Sumatera Utara Tahun Masuk 2008. Skripsi. Medan: Fakultas Kedokteran Universitas Sumatera Utara (2011).

[29] Ward Priestman, Tony Anstis, Isabel G Sebire, Shankar Sridharan, and Neil J Sebire. 2019. Phishing in healthcare organisations: Threats, mitigation and approaches. BMJ health \& care informatics 26, 1 (2019).

[30] Eduardo Remor. 2006. Psychometric properties of a European Spanish version of the Perceived Stress Scale (PSS). The Spanish journal of psychology 9, 1 (2006), 86.

[31] Charles Spearman. 1961. The proof and measurement of association between two things. (1961)

[32] Katrin Starcke and Matthias Brand. 2012. Decision making under stress: a selective review. Neuroscience \& Biobehavioral Reviews 36, 4 (2012), 1228-1248.

[33] M Szalavitz. 2012. Decision making under stress: The brain remembers rewards, forgets punishments. Time.

[34] Keith S Taber. 2018. The use of Cronbach's alpha when developing and reporting research instruments in science education. Research in Science Education 48, 6 (2018), 1273-1296.

[35] Mohsen Tavakol and Reg Dennick. 2011. Making sense of Cronbach's alpha. International journal of medical education 2 (2011), 53.

[36] Evangelia Tsiga, Efharis Panagopoulou, and Anthony Montgomery. 2017. Examining the link between burnout and medical error: A checklist approach. Burnout Research 6 (2017), 1-8.

[37] UiO. 2010. Hva er Nettskjema. Retrieved May 25, 2021 from http://www.uio.no/ tjenester/it/applikasjoner/nettskjema/mer-om/

[38] Verizon. 2021. 2021 data breach investigations report. Retrieved May 24, 2021 from https://enterprise.verizon.com/resources/reports/dbir

[39] Merrill Warkentin and Robert Willison. 2009. Behavioral and policy issues in information systems security: the insider threat. European fournal of Information Systems 18, 2 (2009), 101-105.

[40] Annalena Welp, Laurenz L Meier, and Tanja Manser. 2015. Emotional exhaustion and workload predict clinician-rated and objective patient safety. Frontiers in psychology 5 (2015), 1573

[41] Stephanie E Wemm and Edelgard Wulfert. 2017. Effects of acute stress on decision making. Applied psychophysiology and biofeedback 42, 1 (2017), 1-12.

[42] Monica Whitty, James Doodson, Sadie Creese, and Duncan Hodges. 2015. Individual differences in cyber security behaviors: an examination of who is sharing passwords. Cyberpsychology, Behavior, and Social Networking 18, 1 (2015), 3-7.

[43] Zheng Yan, Thomas Robertson, River Yan, Sung Yong Park, Samantha Bordoff, Quan Chen, and Ethan Sprissler. 2018. Finding the weakest links in the weakest link: How well do undergraduate students make cybersecurity judgment? Computers in Human Behavior 84 (2018), 375-382.

[44] Prosper Yeng, Bian Yang, and Einar Snekkenes. 2019. Observational Measures for Effective Profiling of Healthcare Staffs' Security Practices. In 2019 IEEE 43rd Annual Computer Software and Applications Conference (COMPSAC), Vol. 2. IEEE, 397-404.

[45] Prosper Kandabongee Yeng, Bian Yang, and Einar Arthur Snekkenes. 2019. Framework for Healthcare Security Practice Analysis, Modeling and Incentivization. In 2019 IEEE International Conference on Big Data (Big Data). IEEE, 3242-3251. 